\title{
Unitariness, evidence and quality in bio-medicolegal sciences
}

\author{
Santo Davide Ferrara $\cdot$ Heidi Pfeiffer
}

Received: 1 June 2010 / Accepted: 1 June 2010 / Published online: 16 June 2010

(C) Springer-Verlag 2010

The "global society of risk" in today's post-modern world is increasingly permeated with scientific-technological content, heralding uncertainty and weakness due to the high frequency of errors, whether systematic, erratic, casual, true or false, unintentional or, more rarely, intentional.

The so-called incontrovertible truths flowing into the "cemetery of errors" of science are de-structured and re-structured depending on a "contradictory" and "erratic" periodicity.

The myth of the supremacy and infallibility of science, long trusted by juridical science and jurisprudence, has vanished, being dealt the final blow by the outcome of the pervasive Daubert vs. Merrel Dow Pharmaceuticals sentence of the U.S.A. Supreme Court (509 U.S. 579, 1993).

In U.S. and European criminal and civil laws, the "juridical construction of science", entailing scientific knowledge by hypothesis, contingently true, acquires validity in relation to the specific aim pursued. And in which, for the sciences of risk (including biomedical sciences), general and specific causality is affirmed or negated depending on the rate of error and probability. That is, principles of cause and causality, error rates, theories on

S. D. Ferrara $(\bowtie)$

Forensic Toxicology and Antidoping,

University-Hospital of Padova,

Via Falloppio 50,

35121 Padova, Italy

e-mail: santodavide.ferrara@unipd.it

\section{H. Pfeiffer $(\square)$}

Institut für Rechtsmedizin, Westfälische Wilhelms-Universität,

Röntgenstrasse 23,

48149 Münster, Germany

e-mail: pfeiffh@uni-muenster.de "probability" and "relativistic truths", innate to the history of thought and philosophy, are adopted to fit pre-chosen theoretical systems.

In this framework, the evaluative epi-crises and the conclusions of the "biomedical sciences of risk" pertain to a system of knowledge, the reliability, truth or falsity of which depends on the contemporary "transitory theoretical-practical systematics" of biomedicine, whose progress relies upon the discovery of errors and the processing of new theories. This confirms the Hippocratic Oath of the Third Millennium, which founded the ethical role of the physician on his professionalism, or rather, on the capacity to play the role of researcher, in the constant search for errors, the discovery of which reduces uncertainty in science, enhances professional formation and improves the quality of the system.

In the context of the biomedical sciences, the paramount goal of legal medicine is "to discover the truth and state the justice", this being achieved by applying proper, particular "SYSTEMATICS". This, declinable as "methodology of ascertainment" and "criteriology of evaluation", aims at: acquisition of the objectivity of the datum, and its translation into proof, if possessing the characteristics of evidence and irrefutability; epi-critical interpretation, aiming for evaluative conclusions of a diverse nature and finality.

"Systematics" capable of encompassing and unifying multiple budding, whose gradual innovation and sometimes pervasive extension has led to the generation, the development and the definitive consolidation of disciplines such as forensic pathology, forensic genetics, forensic toxicology, forensic psychopathology, criminology and forensic anthropology, as well as that of many other sectors of study, to indepth investigation, that have yet to be consolidated in further disciplines.

This tumultuous disciplinary budding process has led to a loss of unitariness in medicolegal knowledge, with an 
incurrent risk of falling into an irreversible fragmentation and the impoverishment of the same knowledge, no longer nourished by cultural products of high interdisciplinary value. It is evident, in fact, that knowledge is enriched not through highly specialized sophistication, but also, and above all, through interdisciplinarity.

If the process underway is thus represented, and if it remains as such in the future, legal medicine, apart from risking exclusion from innovation, will risk incurring the definitive and irreversible loss of its own unity of knowledge.

The above issue cannot be taken lightly for it is of vital importance: on it hinges the very future and survival of the medicolegal discipline, whose priority ethicality must include the preservation of the unitariness and the development of evidence and quality.

Notwithstanding the difficulty intrinsic to definition, measurement and demonstration, quality in medicine in general and the "quality of the medicolegal system" in particular are attainable via the valorization of the work of the individual professional within an integrated vision and system capable of translating the concept of quality to every-day practice.

Therefore a quality system, founded on "continuous disciplinary education", must move towards achieving the objectives of "accuracy, efficacy, timeliness and efficiency", evaluated by applying "internal control" and "external proficiency programmes", and must strive for the "certification" and "accreditation" of "institutions" and individual "professionals".

Continuous education, systems of control and proficiency, certification and accreditation hinge upon guidelines and protocols shared and used in the circle of the scientific and professional community of reference.

A renewed commitment, oriented towards a "logicalevaluative process" sustained by "guidelines", "scientific documentation" and "reference frames inherent to criteria", but also oriented towards changing operational practice, is to be considered mandatory in bio-medicolegal sciences, as a key to satisfying the needs of "evidence-based medicine" and to keeping in line with post-modernity.

The course of the above process is confirmed by the cognitive and operative objectives defined in the article "Bio-medicolegal guidelines and protocols. Survey and future perspectives in Europe" in the current issue of the International Journal of Legal Medicine [1]. The preliminary findings made in this study demonstrate that interdisciplinary synergy and a supranational perspective are adequate resources in the future preservation, development, innovation and unitariness of bio-medicolegal learning ... in step with post-modern times.

The journal, the official organ of the International and European Academy of Legal Medicine, in finalizing its own work of divulgation of scientific and professional knowledge, re-proposes itself as co-protagonist in the process of disciplinary development and innovation for the benefit of the values of truth and justice that underpin medicolegal goals.

\section{Reference}

1. Ferrara SD, Viel G, Case C, Snenghi R, Cecchi R, Bajanowski T (2010) Bio-medicolegal guidelines and protocols. Survey and future perspectives in Europe. Int J Leg Med 124:345-350 